\title{
The roles of triglyceride/high-density lipoprotein cholesterol ratio and uric acid as predisposing factors for metabolic syndrome in healthy children
}

Maria Efthymia Katsa, MSc, $\mathrm{PhD}^{1}$, Anastasios loannidis, MD, $\mathrm{PhD}^{1}$, Athanasios Sachlas, $\mathrm{PhD}^{2}$, loannis Dimopoulos, $\mathrm{PhD}^{3}$, Stylianos Chatzipanagiotou, $\mathrm{MD}, \mathrm{PhD}^{4}$, Andrea Paola Rojas Gil, $\mathrm{PhD}^{1}$

${ }^{1}$ Department of Nursing, Faculty of Health Sciences, University of Peloponnese, Tripolis, Greece

${ }^{2}$ Department of Statistics and Insurance Science, Faculty of Finance and Statistics, University of Piraeus, Athens, Greece

${ }^{3}$ School of Management, University of Peloponnese, Kalamata, Greece

${ }^{4}$ Department of Biopathology and Clinical Microbiology, Aeginition Hospital, Kapodistrian University of Athens Medical School, Athens, Greece

Received: 19 October, 2018 Revised: 10 December, 2018 Accepted: 3 February, 2019

Address for correspondence: Andrea Paola Rojas Gil, PhD

Department of Nursing, Faculty of Health Sciences, University of Peloponnese, Dept. of Economics Building 2nd floor, Sehi area, Tripoli 22100, Greece

Tel: +2710230125

Fax:+2710230174

E-mail: arojas@uop.gr,apaola71@ yahoo.com.mx

https://orcid.org/0000-0002-98122131
Purpose: To estimate the roles of triglyceride/high-density lipoprotein cholesterol (TG/HDL) ratio and uric acid in predisposition for metabolic syndrome (MetS) and its components in healthy children.

Methods: Anthropometric and biochemical analyses were performed on 110 children, aged 5 to 12 years, from the Greek county of Laconia. The children were studied as a whole population and in separate groups according to age and predisposition to MetS after taking into consideration International Diabetes Federation criteria, body mass index, and lipid profile.

Results: Seventeen percent of children exhibited predisposition to MetS, while $39.1 \%$ had TG/HDL ratio $>1$, and $3.64 \%$ had high level of uric acid. According to a receiver operating characteristic curve analysis, the relative probability for MetS predisposition sextupled when TG/HDL ratio was $\geq 1$ (odds ratio [OR], 5.986; 95\% confidence interval $[\mathrm{Cl}], 1.968-18.205)$. Children in the total population and those aged $<9$ years had a greater probability for increased low-density lipoprotein (LDL) cholesterol (OR, 3.614; $95 \% \mathrm{Cl}, 1.561-8.365)$ when TG/HDL ratio was $\geq 1$. The TG/HDL ratio was positively correlated with body mass index $(\mathrm{BMI})(P=0.035)$ in children without MetS, cholesterol in the total population $(P=0.06)$ and children $\geq 9$ years old $(P=0.026)$, and with $L D L$ in the total population and both age groups $(P=0.001)$. The $\mathrm{TG} / \mathrm{HDL}$ ratio was also positively correlated with alanine aminotransferase in the total population ( $P=0.033$ ) and gamma-glutamyl transferase in most studied groups $(P<0.001)$. Uric acid was positively correlated with waist circumference in the total population $(P=0.043)$ and in those without MetS $(P=0.027)$. It was also positively correlated with $\mathrm{BMI}, \mathrm{TG}$, cholesterol, and TG/HDL ratio and negatively correlated with HDL in most studied groups $(P<0.005)$.

Conclusion: The studied parameters correlated with MetS components and could be characterized as effective indexes for childhood MetS, regardless of age and predisposition to MetS.

Keywords: Triglyceride/high-density lipoprotein cholesterol ratio, Uric acid, Metabolic syndrome, Child

\section{Introduction}

Metabolic syndrome (MetS) consists of 6 components that probably lead to type 2 diabetes mellitus and cardiovascular disease: central fat adiposity, hypertension, atherogenic dyslipidemia, glucose intolerance, a proinflammatory state, and a prothrombotic state. ${ }^{1)}$ These disturbances may occur in early childhood and persist into adulthood. ${ }^{2)}$

In MetS, hypertriglyceridemia, as a part of atherogenic dyslipidemia, causes a reduction in high-density lipoprotein (HDL) cholesterol level and an increase in small, low-density 
lipoprotein (LDL) particles, leading to high risk of myocardial infarction. ${ }^{3)}$ HDL cholesterol plays a protective role against atherogenic procedures by delivering excess cholesterol to the liver, inhibiting LDL oxidation, arousing prostacyclin secretion, and decreasing platelet aggregation. ${ }^{4)}$ Previous studies indicate that it is not always possible to determine the increase of LDL particle size, which is a cause of hypetriglyridemia. ${ }^{3)}$ For that reason, triglyceride (TG)/HDL ratio has been considered a useful and accurate marker of atherogenicity. ${ }^{3,5)}$ Liver and muscle tissue quantities also play crucial roles in development of diabetes mellitus. The TG/HDL ratio could be considered a marker for insulin resistance.

Uric acid is also correlated with development of MetS. ${ }^{6,7)}$ It is an antioxidant and plays a compensatory role in oxidative stress, such as stress associated with coronary disease. ${ }^{6}$ ) Despite its antioxidant role in the extracellular environment, uric acid has a detrimental impact in intracellular locations and is predictive of complications associated with MetS, including diabetes mellitus, hypertension, and renal dysfunction. ${ }^{7,8)}$

Uric acid and TG/HDL ratio are not included in International Diabetes Federation (IDF) criteria for MetS, despite both TG and HDL included as individual components of the criteria. ${ }^{9)}$ However, TG/HDL ratio and uric acid appear to play key roles in metabolic disorders. In obese children, TG/HDL ratio may be characterized as a predisposing factor for MetS and its components. ${ }^{10-12)}$ Similarly, uric acid plays a contributing role in development of childhood MetS. ${ }^{13-17)}$ While most studies of the roles of uric acid and TG/HDL have involved only adults, some research has been performed over the last 5 years on children and adolescent populations. More emphasis is given to obese children, especially with respect to the role of TG/HDL ratio.

Given the lack of knowledge, the aim of the present study was to investigate the correlations of TG/HDL ratio and uric acid with MetS and its components, taking into account age and predisposition to MetS.

\section{Materials and methods}

\section{Studied population and ethics}

The current survey was conducted in the Prefecture of Laconia, Greece, under the "Prevention of childhood obesity and type 2 diabetes mellitus" educational program. The survey was approved by the Hellenic Ministry of Education, Department of Healthcare and Environmental Education (No 6884/67), according to the provisions of the Helsinki declaration of 1975 , as revised in 1983 .

A total of 110 children volunteers aged 5 to 12 years was enrolled in the study. Children were randomly selected from 8 of the 18 primary schools and kindergartens in Laconia. Multistage stratified sampling was conducted. Parents and/ or guardians provided informed consent for anthropometric measurements and biochemical analysis. Children who were systematically taking medication, children with diagnosed chronic disease, and children with premature adrenarche were excluded from the study. Anonymity of the school, parents, and children was assured. No personal data were collected. The sample size was sufficient to evaluate standardized differences greater than 0.5 between the investigated parameters, achieving statistical power $>0.80$ at a $P<0.05$.

\section{Anthropometric characteristics}

Height, weight, waist circumference (WC), and hip circumference (HC) of all participants were measured. Body mass index (BMI) and WC were calculated in accordance with each child's age and sex. ${ }^{18)} \mathrm{HC} / \mathrm{WC}$ ratio was also calculated. Blood pressure was measured twice for each child, with a 10-minute break between measurements. The final value used for further analysis for blood pressure was the mean of the 2 measurements.

\section{Laboratory measures, blood samples, and serum assays}

Peripheral blood samples from 110 fasting children were gathered. Complete biochemical tests were performed for blood lipid profile, fasting blood glucose (FBG), and markers of liver function. Serum analyses were conducted using the same procedure and at the same lab with the use of a biochemical analyzer (Olympus AU600, Olympus, Tokyo, Japan). TG/HDL and cholesterol/HDL ratios, which indicate the risk for coronary artery disease (CAD), were also calculated. CAD as an index has high sensitivity and specificity for cardiovascular disease risk factors-higher than that of cholesterol or HDL separately. ${ }^{19)}$

\section{Diagnostic criteria for MetS predisposition}

MetS can be characterized as a combination of 3 or more components of obesity, abnormal glucose homeostasis, hypertension, and dyslipidemia. ${ }^{20)}$ In the current study, MetS diagnoses were based on IDF criteria ${ }^{9}$ in combination with endothelial dysfunction risk factors of BMI $\leq 85$ th percentile, fasting $\mathrm{LDL} \leq 100 \mathrm{mg} / \mathrm{dL}$, and total cholesterol $\leq 200 \mathrm{mg} /$ $\mathrm{dL}^{2,21,22)}$ Consequently, in this study, a child was characterized as predisposed for MetS when 3 or more factors from the following coexisted: increased WC\%, BMI\%, FBG, fasting TG, blood pressure, fasting LDL, and total cholesterol and decreased fasting HDL.

\section{Studied categories}

The onset of puberty in females is defined as the beginning of breast development and in males as testicular volume $>3 \mathrm{~mL}$. These changes typically occur after the age of 8 years in girls and 9 in boys. In Greece, the mean age of pubertal onset has been reported at approximately 10 years. Metabolic parameters appear to be affected by onset of puberty. The current study considered an average age of 9 years for puberty onset to study the effects on metabolism. Specifically, children were placed in 
4 subcategories according to age and predisposition to MetS. Specifically, children were studied as a whole population and in the following subcategories: children with $(\mathrm{n}=19)$ or without $(\mathrm{n}=91)$ predisposition for MetS and children aged $<9(\mathrm{n}=51)$ years or $\geq 9$ years $(n=59)$ after taking into consideration the approximate age of tanner stage 2 onset. $^{23,24)}$

After studying these subcategories, the independent impacts of obesity and central fat deposition on TG/HDL and uric acid roles were also investigated by studying separately overweight children, children with central obesity, and children with normal BMI $\left(\mathrm{kg} / \mathrm{m}^{2}\right)$ and WC.

\section{Statistical analysis}

Pearson correlation was used to evaluate the relationships between quantitative variables. Logistic regression analysis was performed; specifically, odds ratios (ORs) were calculated to measure the associations between categorical variables. Statistical analysis was performed using IBM SPSS Statistics ver. 24.0 (IBM Co., Armonk, NY, USA), with a significance level set at $5 \%$.

\section{Results}

\section{Studied population}

Boys and girls were equally distributed (50\% for each group). Slightly more than half $(53.6 \%)$ of the children were $\geq 9$ years old. A total of $39.1 \%$ had $\mathrm{BMI} \% \geq 85$, and $71.8 \%$ had a WC $\% \geq 95$. Of the studied population, $17.27 \%$ was predisposed to MetS (with 3 or more predisposition parameters). Table 1 depicts baseline the biochemical and anthropometric characteristics of all studied subcategories.

\section{TG/HDL ratio as a predisposing factor for MetS}

The TG/HDL ratio was $>1$ in $39.1 \%$ of the participants $(43$ children): $2: 1$ in $3.6 \%$ of the participants ( 4 children), $3: 1$ in $1.8 \%$ ( 2 children), and $4: 1$ in $1.8 \%$ ( 2 children).

Receiver operating characteristic curve (ROC) analysis showed that TG/HDL ratio had high sensitivity (73.7\%) and specificity $(68.1 \%)$ for MetS predisposition with a cutoff point of 1 (specifically 1.0082). The area under the curve was 0.722 (95\% confidence interval [CI], 0.595-0.849), which indicates diagnostic accuracy. The percentage of correct classification was 69.1 .

The relative probability (odds for diagnosing a child with predisposition) for MetS when the TG/HDL ratio was greater than 1 was almost 6 times greater than the relative probability (odds) when TG/HDL ratio was less than 1 (OR, 5.986; 95\% CI, 1.968-18.205).

\section{Correlations between TG/HDL ratio, anthropometric characteristics, and biochemical markers}

The analysis was performed in all subcategories as mentioned

Table 1. Mean values of the baseline characteristics in all studied categories $(n=110)$

\begin{tabular}{|c|c|c|c|c|c|c|}
\hline Characteristic & $\begin{array}{l}\text { Children with } \\
\text { predisposition for } \\
\text { MetS }(n=19)\end{array}$ & $\begin{array}{l}\text { Children without } \\
\text { predisposition for } \\
\text { MetS }(n=91)\end{array}$ & $P$-value & $\begin{array}{c}\text { Children }<9 \text { years } \\
\text { old }(n=51)\end{array}$ & $\begin{array}{c}\text { Children } \geq 9 \text { years } \\
\text { old }(n=59)\end{array}$ & $P$-value \\
\hline $\mathrm{HDL}$ & $66 \pm 13.9$ & $66.98 \pm 11.9$ & 0.753 & $68 \pm 11.1$ & $65.6 \pm 13$ & 0.275 \\
\hline LDL & $117.5 \pm 15.6$ & $88.7 \pm 16.1$ & $<0.001$ & $91 \pm 17.9$ & $93 \pm 20.6$ & 0.753 \\
\hline TG & $96.3 \pm 51.8$ & $58.2 \pm 23.7$ & 0.297 & $60.4 \pm 33$ & $65.5 \pm 23.6$ & 0.354 \\
\hline Cholesterol & $196.47 \pm 19.9$ & $157.3 \pm 21.9$ & 0.001 & $165.2 \pm 23.5$ & $163.8 \pm 28.4$ & 0.633 \\
\hline FBG & $86.2 \pm 4.1$ & $85.95 \pm 6.25$ & 0.860 & $85.1 \pm 5.96$ & $86.8 \pm 5.9$ & 0.129 \\
\hline Uric acid & $3.29 \pm 1.05$ & $3.37 \pm 0.93$ & 0.749 & $2.99 \pm 0.82$ & $3.67 \pm 0.9$ & $<0.001$ \\
\hline ALT/SGPT & $20.58 \pm 5.82$ & $16.7 \pm 2.3$ & 0.001 & $17.6 \pm 5.8$ & $17.2 \pm 6.2$ & 0.748 \\
\hline AST/SGOT & $25.3 \pm 5.36$ & $26.5 \pm 6.99$ & 0.478 & $27.68 \pm 6$ & $25.2 \pm 7.16$ & 0.053 \\
\hline ALP & $278.5 \pm 46$ & $264,8 \pm 64.1$ & 0.376 & $254.1 \pm 47.6$ & $278.3 \pm 70$ & 0.035 \\
\hline GGT & $18.53 \pm 2.17$ & $16.63 \pm 2.3$ & 0.001 & $16.54 \pm 2.3$ & $17.3 \pm 2.4$ & 0.096 \\
\hline CAD (cholesterol/HDL ratio) & $3.09 \pm 0.72$ & $2.39 \pm 0.39$ & $<0.001$ & mean: $2.48 \pm 0.5$ & mean: $2.55 \pm 0.57$ & 0.492 \\
\hline TG/HDL ratio & $1.47 \pm 1.06$ & $0.925 \pm 0.48$ & 0.297 & $0.95 \pm 0.68$ & $1.3 \pm 1.6$ & 0.160 \\
\hline $\mathrm{BMI} \%$ & $81.5 \pm 23.8$ & $58.7 \pm 32.29$ & 0.004 & $63.9 \pm 33.8$ & $61.8 \pm 30.9$ & 0.735 \\
\hline BMI z-score & $0.81 \pm 1.18$ & $0.48 \pm 1.2$ & 0.308 & $0.75 \pm 1.04$ & $0.38 \pm 1.3$ & 0.095 \\
\hline WC\% & $95 \pm 0$ & $83 \pm 21.6$ & 0.023 & $86.89 \pm 21.2$ & $85.3 \pm 19.3$ & 0.888 \\
\hline HC/WC & $1.17 \pm 0.17$ & $1.13 \pm 0.086$ & 0.079 & $1.12 \pm 0.114$ & $1.12 \pm 0.156$ & 0.836 \\
\hline SBP\% & $120 \pm 12.4$ & $111.6 \pm 15.9$ & 0.029 & $106.2 \pm 18.4$ & $118.2 \pm 10.9$ & $<0.001$ \\
\hline
\end{tabular}

Values are presented as mean \pm standard deviation.

HDL, high-density lipoprotein; LDL, low-density lipoprotein; TG, triglyceride; FBG, fasting blood glucose; ALT/SGPT, alanine aminotransferase/serum glutamic-pyruvic transaminase; AST/SGOT, aspartate aminotransferase/serum glutamic-oxaloacetic transaminase; ALP, alkaline phosphatase; GGT, gamma-glutamyl transferase; CAD, coronary artery disease; BMI, body mass index; WC, waist circumference; HC, hip circumference; SBP, systolic blood pressure. 
above. Table 2 depicts briefly the correlations with TG/HDL ratio.

1) TG/HDL ratio and anthropometric characteristics

TG/HDL ratio was positively correlated with $\mathrm{BMI} \%(P=0.035)$ in children without predisposition for MetS.

2) TG/HDL ratio and lipid profile

TG/HDL ratio was positively correlated with cholesterol $(P=0.06)$ in the total population and in children aged $\geq 9$ years

Table 2. Correlation analysis study between TG/HDL ratio, biochemical markers and anthropometric characteristics in all studied categories

\begin{tabular}{|c|c|c|c|c|c|}
\hline \multirow[b]{2}{*}{ Parameter } & \multicolumn{5}{|c|}{ TG/HDL ratio } \\
\hline & $\begin{array}{l}\text { Total population } \\
\qquad(n=110)\end{array}$ & $\begin{array}{l}\text { Children with } \\
\text { Predisposition for } \\
\text { MetS ( }=19)\end{array}$ & $\begin{array}{c}\text { Children without } \\
\text { Predisposition for } \\
\text { MetS ( }=91)\end{array}$ & $\begin{array}{l}\text { Children }<9 \text { years old } \\
\qquad(n=51)\end{array}$ & $\begin{array}{l}\text { Children } \geq 9 \text { years old } \\
\qquad(n=59)\end{array}$ \\
\hline $\mathrm{HDL}$ & $P<0.001, r=-0.449$ & $P=0.008, r=-0.592$ & $P<0.001, r=-0.661$ & $P<0.001, r=-0.611$ & $P<0.001, r=-0.414$ \\
\hline LDL & $P=0.001, r=0.326$ & NS & NS & $P=0.01, r=0.362$ & $P=0.006, r=0.354$ \\
\hline TG & $P<0.001, r=0.946$ & $P<0.001, r=0.965$ & $P<0.001, r=0.935$ & $P<0.001, r=0.977$ & $P<0.001, r=0.891$ \\
\hline Cholesterol & $P=0.006, r=0.258$ & NS & NS & NS & $P=0.026, r=0.290$ \\
\hline FBG & NS & NS & NS & NS & NS \\
\hline Uric acid & NS & NS & $P=0.002, r=0.316$ & $P=0.017, r=0.335$ & NS \\
\hline ALT/SGPT & $P=0.033, r=0.204$ & NS & NS & NS & NS \\
\hline AST/SGOT & NS & NS & NS & NS & NS \\
\hline ALP & NS & NS & NS & NS & NS \\
\hline GGT & $P<0.001, r=0.331$ & $P=0.002, r=0.658$ & NS & NS & $P=0.002, r=0.390$ \\
\hline CAD (cholesterol/HDL ratio) & $P<0.001, r=0.728$ & $P<0.001, r=0.831$ & $P<0.001, r=0.665$ & $P<0.001, r=0.768$ & $P<0.001, r=0.765$ \\
\hline BMI\% & NS & NS & $P=0.035, r=0.221$ & NS & NS \\
\hline WC\% & NS & NS & NS & NS & NS \\
\hline $\mathrm{HC}$ & $P=0.041, r=0.195$ & NS & NS & $P=0.032, r=0.304$ & NS \\
\hline HC/WC & NS & NS & NS & NS & NS \\
\hline
\end{tabular}

HDL, high-density lipoprotein; LDL, low-density lipoprotein; TG, triglyceride; FBG, fasting blood glucose; ALT/SGPT, alanine aminotransferase/serum glutamic-pyruvic transaminase; AST/SGOT, aspartate aminotransferase/serum glutamic-oxaloacetic transaminase; ALP, alkaline phosphatase; GGT, gamma-glutamyl transferase; CAD, coronary artery disease; BMI, body mass index; WC, waist circumference; HC, hip circumference; NS, nonsignificant.

Table 3. Correlation analysis study between uric acid, biochemical markers and anthropometric characteristics in all studied categories

\begin{tabular}{|c|c|c|c|c|c|}
\hline \multirow[b]{2}{*}{ Parameter } & \multicolumn{5}{|c|}{ Uric acid } \\
\hline & $\begin{array}{l}\text { Total population } \\
\qquad(n=110)\end{array}$ & $\begin{array}{l}\text { Children with } \\
\text { Predisposition for } \\
\text { MetS }(n=19)\end{array}$ & $\begin{array}{l}\text { Children without } \\
\text { Predisposition for } \\
\text { MetS }(n=91)\end{array}$ & $\begin{array}{l}\text { Children }<9 \text { years old } \\
\qquad(n=51)\end{array}$ & $\begin{array}{l}\text { Children } \geq 9 \text { years old } \\
\qquad(n=59)\end{array}$ \\
\hline $\mathrm{HDL}$ & $P<0.001, r=-0.448$ & $P=0.012, r=-0.562$ & $P<0.001, r=-0.431$ & $P=0.026, r=-0.316$ & $P<0.001, r=-0.517$ \\
\hline LDL & NS & NS & NS & NS & NS \\
\hline $\mathrm{TG}$ & $P=0.008, r=0.255$ & $P=0.002, r=0.689$ & NS & $P=0.038, r=0.295$ & NS \\
\hline Cholesterol & NS & NS & NS & NS & NS \\
\hline FBG & NS & NS & NS & NS & NS \\
\hline TG/HDL ratio & NS & NS & $P=0.002, r=0.316$ & $P=0.017, r=0.335$ & NS \\
\hline ALT/SGPT & NS & NS & NS & NS & NS \\
\hline AST/SGOT & NS & NS & NS & NS & NS \\
\hline ALP & $P=0.036, r=0.201$ & NS & NS & NS & NS \\
\hline GGT & $P=0.037, r=0.199$ & NS & NS & NS & NS \\
\hline CAD (cholesterol/HDL ratio) & $P=0.006, r=0.259$ & $P=0.037, r=0.480$ & $P=0.008, r=0.278$ & $P=0.029, r=0.310$ & NS \\
\hline BMI\% & $P<0.001, r=0.429$ & $P=0.007, r=0.596$ & $P<0.001, r=0.417$ & NS & $P=0.01, r=0.332$ \\
\hline WC\% & $P=0.043, r=0.199$ & NS & $P=0.027, r=0.240$ & NS & NS \\
\hline $\mathrm{HC}$ & $P=0.01, r=0.243$ & $P=0.007, r=0.601$ & $P=0.037, r=0.219$ & NS & NS \\
\hline HC/WC & $P=0.025, r=-0.214$ & NS & NS & NS & NS \\
\hline
\end{tabular}

HDL, high-density lipoprotein; LDL, low-density lipoprotein; TG, triglyceride; FBG, fasting blood glucose; ALT/SGPT, alanine aminotransferase/serum glutamic-pyruvic transaminase; AST/SGOT, aspartate aminotransferase/serum glutamic-oxaloacetic transaminase; ALP, alkaline phosphatase; GGT, gamma-glutamyl transferase; CAD, coronary artery disease; BMI, body mass index; WC, waist circumference; HC, hip circumference; NS, nonsignificant. 
$(P=0.026)$. It was also positively correlated with LDL in the total population $(P=0.001)$ and in children of both age groups $(P=0.01)$. TG/HDL ratio was positively correlated with CAD $(P<0.001)$ in all studied categories.

According to logistic regression analysis, children had a greater relative probability of having higher LDL when TG/ HDL ratio was $\geq 1$. Specifically, the odds for increased LDL ( $>100 \mathrm{mg} / \mathrm{dL}$ ) were 3.6 times greater for children in the total population (OR, 3.614; 95\% CI, 1.561-8.365) and 8 times greater for children aged $<9$ years (OR, 8.000; 95\% CI, 2.062-31.041).

\section{3) TG/HDL ratio and liver enzymes}

TG/HDL ratio was positively correlated with alanine aminotransferase (ALT/SGPT) $(P=0.033)$ in the total population. Additionally, it was positively correlated with gammaglutamyl transferase $(\mathrm{GGT})(P<0.001)$ in the total population, in children predisposed to MetS $(P=0.002)$, and in children $\geq 9$ years old $(P=0.002)$.

\section{Correlations between uric acid, anthropometric characteristics, and biochemical markers}

Analysis was performed in all subcategories mentioned above. Table 3 summarizes the correlations with uric acid.

1) Uric acid and anthropometric characteristics

A positive correlation between uric acid and BMI was found in the total population $(P<0.01)$, in children with $(P=0.007)$ and without $(P<0.001)$ predisposition for MetS, and in children $\geq 9$ years old $(P=0.01)$. Uric acid was also positively correlated with
WC in the total population $(P=0.043)$ and in children without predisposition for MetS $(P=0.027)$. Finally, uric acid was negatively correlated with $\mathrm{HC} / \mathrm{WC}$ ratio in the total population $(P=0.025)$.

2) Uric acid and lipid profile

Uric acid was positively correlated with triglycerides in the total population $(P=0.008)$, in children with predisposition for $\operatorname{Met} S(P=0.002)$, and in children younger than 9 years $(P=0.038)$. It was also positively associated with $C A D$ in the total population $(P=0.006)$, in children with $(P=0.037)$ and without $(P=0.008)$ predisposition for MetS, and in children younger than $9(P=0.029)$. A negative correlation between uric acid and HDL was found in all studied categories $(P<0.001)$. Moreover, it was positively correlated with TG/HDL ratio in children without predisposition for Met $S(P=0.002)$ and in children younger than $9(P=0.017)$.

3) Uric acid and liver enzymes

In the total population, uric acid was positively correlated with GGT $(P=0.037)$ and alkaline phosphatase (ALP) $(P=0.036)$.

\section{TG/HDL ratio and uric acid in correlation to BMl and WC}

To investigate the roles of obesity and central obesity, children were studied separately according to BMI\% $(<85 \%$ or $\geq 85 \%)$ and WC\% ( $<95 \%$ or $\geq 95 \%)$. No statistically important correlation found regarding TG/HDL ratio and the studied parameters. However, uric acid appeared to have some statistically important correlations, which are listed in Table 4.

Table 4. Correlation analysis study between uric acid, biochemical markers and anthropometric characteristics in children categorized according to their BMI\% and WC\%.

\begin{tabular}{|c|c|c|c|c|}
\hline \multirow[b]{2}{*}{ Parameter } & \multicolumn{4}{|c|}{ Uric acid } \\
\hline & $\begin{array}{c}\text { Children with BMI\% } \% 85 \\
(\mathrm{n}=67)\end{array}$ & $\begin{array}{c}\text { Children with BMI\% } \% 85 \\
\qquad(n=43)\end{array}$ & $\begin{array}{l}\text { Children with WC\% } \% 95 \\
(\mathrm{n}=31)\end{array}$ & $\begin{array}{l}\text { Children with WC\% } \mathrm{x} 95 \\
\qquad(\mathrm{n}=79)\end{array}$ \\
\hline$\overline{\mathrm{HDL}}$ & $r=-0.388, P=0.005$ & $r=-0.006, P<0.001$ & $r=-0.353, P=0.041$ & $r=-0.045, P<0.001$ \\
\hline LDL & NS & NS & NS & NS \\
\hline $\mathrm{TG}$ & NS & $r=0.376, P=0.013$ & NS & $r=0.285, P=0.013$ \\
\hline Cholesterol & NS & NS & NS & NS \\
\hline FBG & NS & NS & NS & NS \\
\hline TG/HDL ratio & NS & NS & NS & NS \\
\hline ALT/SGPT & NS & NS & NS & NS \\
\hline AST/SGOT & NS & NS & NS & NS \\
\hline ALP & $r=0.376, P=0.024$ & NS & NS & NS \\
\hline GGT & $r=0.246, P=0.045$ & NS & NS & NS \\
\hline CAD (cholesterol/HDL ratio) & NS & $r=0.374, P=0.001$ & NS & $r=0.246, P=0.032$ \\
\hline BMI\% & NS & NS & NS & NS \\
\hline WC\% & $r=0.244, P=0.049$ & NS & NS & NS \\
\hline $\mathrm{HC}$ & NS & NS & NS & NS \\
\hline HC/WC & NS & NS & NS & NS \\
\hline
\end{tabular}

BMI, body mass index; WC, waist circumference; HDL, high-density lipoprotein; LDL, low-density lipoprotein; TG, triglyceride; FBG, fasting blood glucose; ALT/SGPT, alanine aminotransferase/serum glutamic-pyruvic transaminase; AST/SGOT, aspartate aminotransferase/serum glutamic-oxaloacetic transaminase; ALP, alkaline phosphatase; GGT, gamma-glutamyl transferase; CAD, coronary artery disease; HC, hip circumference; NS, nonsignificant. 


\section{Discussion}

The aim of this study was to estimate how TG/HDL ratio and uric acid are associated with biochemical markers and anthropometric characteristics in healthy children aged 5 to 12 . Both parameters were positively correlated with a congested lipid profile, obesity, and visceral fat deposition from early childhood, predisposing them to MetS and its complications.

Because our previous study determined that lifestyle habits have more beneficial effects in children without MetS, ${ }^{2)}$ we investigated if TG/HDL ratio and uric acid have special roles in metabolic parameters according to predisposition for MetS.

Previous research has shown that high TG/HDL $(>4)$ is an important and independent predictor of coronary disease in adults. ${ }^{25)}$ Regarding obese children and adolescents, TG/ HDL ratio has high sensitivity (80\%) and specificity (75\%) for MetS with a cutoff point of $1.25{ }^{10)}$ The present study also showed through an ROC analysis that a ratio $>1$ sextuples the possibility of predisposition to MetS, despite the normal ranges of triglycerides and HDL as 2 single factors, in the majority of children. This indicates that children are at high risk, especially if we take into consideration that $39.1 \%$ of them had TG/HDL ratio $>1$.

In the current survey, TG/HDL ratio was positively correlated with CAD, triglycerides, cholesterol, and LDL and negatively correlated with HDL. Our results expand on previous studies that found a close relationship between TG/HDL ratio and risk of cardiovascular disease. ${ }^{3)}$ Triglycerides are correlated with an increased population of small, dense LDL particles, which enhance the transfer of cholesteryl esters from HDL to apolipoprotein $\mathrm{B}^{26)}$ However, the role of HDL is different; it has an antithrombotic effect, reversing the endothelial cell dysfunction. ${ }^{4)}$ TG/HDL ratio is positively correlated with small, dense LDL particles, which are characterized as atherogenic and predispose patients to cardiovascular disorders. ${ }^{3)}$

The present study identified positive correlation among TG/HDL ratio, BMI, ALT/SGPT, and GGT. It has been established that TG/HDL is associated with increased BMI and WC in children and can leading to metabolic disorders. ${ }^{27}$ Adipose tissue inflammation is involved in development of diabetes and cardiovascular disease, especially in obese people. Insulin resistance is therefore correlated with fatty acid accumulation in the liver, which reduced endogenous liver glucose production. ${ }^{27,28)}$ In addition, TG/HDL ratio has been characterized as a preclinical sign of nonalcoholic fatty liver disease (NAFLD) in children. ${ }^{27)}$

Regarding the role of uric acid in predisposition for MetS and its components, the current survey showed positive correlation with BMI, WC, triglycerides, and CAD and a negative correlation with HDL. It has already been shown that hyperuricemia is positively correlated with MetS. ${ }^{8)}$ Specifically, deposition of visceral fat can aggravate the lipid profile and increase the concentration of insulin through a reduction in renal excretions, resulting in hyperuricemia. ${ }^{13)}$ In addition, increased uric acid is an independent risk factor for cardiovascular disease due to its mechanistic role in atherosclerosis through removal of nitric oxide ${ }^{13)}$ and its relationship with small, dense LDL and HDL cholesterol particles. ${ }^{29,30)}$ A mechanism that explains the correlation between uric acid and triglycerides has not been found, ${ }^{31)}$ although lifestyle and genetics may be crucial. ${ }^{31)}$

In general, uric acid has been characterized as an indicator of triglyceride level. ${ }^{32)}$ In the present study, uric acid also was positively correlated with GGT and ALP. A possible explanation is that secretion of uric acid may have a negative impact, predictive of complications of MetS, due to oxidative stress. ${ }^{7,8)}$ Additionally, oxidative stress and insulin resistance play crucial roles in development of liver diseases. Hyperuricemia, which reflects the procedures of oxidative stress, is therefore thought to be a risk factor for NAFLD. Uric acid level could be characterized as an indicator of liver disease. ${ }^{33)}$

Obesity and central obesity are included in the predisposition parameters for MetS. To investigate the role of these parameters in correlation with TG/HDL ratio and uric acid as aggravating factors for metabolic disorders, statistical analysis was performed categorizing children according to BMI\% and $\mathrm{WC} \%$. This analysis showed only uric acid to have statistically positive correlation with triglycerides and CAD in overweight children and children with central obesity. This observation implies a need for obesity prevention, as it appears to be an independent risk factor for endothelial dysfunction.

According to the IDEFICS (identification and prevention of dietary- and lifestyle-induced health effects in children and infants) cohort, MetS score depicts a positive trend with child age. ${ }^{34)}$ Age also plays a crucial role in NAFLD acquisition, as fatty liver prevalence increases with age. ${ }^{35)}$ The positive correlation of congested lipid profile with MetS can be understood from the MetS components, ${ }^{2,9)}$ while a predisposition may signal the beginning of a vicious circle that exists with NAFLD. ${ }^{36)}$ Taking into consideration the data mentioned above, it was possible to hypothesize that children $\geq 9$ years old or predisposed to MetS could see greater impact on biochemical markers from TG/HDL ratio and uric acid. However, apart from GGT and total cholesterol levels (which were correlated with TG/HDL ratio in older children) and uric acid (which was correlated with TG/HDL ratio in younger children and children without predisposition for MetS), both studied parameters affected anthropometric characteristics and lipid profiles in all studied categories, indicating the importance of both indexes as early indicators for metabolic disorders.

The current study presents some limitations. More children could have participated in the study if their parents or guardians had agreed to participation in biochemical analyses. In addition, Laconia is a Peloponnese county, and the results cannot be generalized to the whole country. Further research is necessary to establish the exact mechanisms of TG/HDL ratio and uric acid action.

In conclusion, the studied parameters appear to be important predisposing factors for metabolic disorders. Specifically, TG/ HDL ratio and uric acid may be early predictors for MetS, as they indicate endothelial dysfunction, predisposition for 
cardiovascular complication, and hepatic dysfunction from early childhood. Age and predisposition for MetS do not appear to differentiate as early predictors.

\section{Ethical statement}

The survey was approved by the Hellenic Ministry of Education, Department of Healthcare and Environmental Education (No. 6884/67), according to the provisions of the Helsinki declaration of 1975, as revised in 1983. Parents and/ or guardians provided informed consent for anthropometric measurements and biochemical analysis.

\section{Conflict of interest}

No potential conflict of interest relevant to this article was reported.

\section{References}

1. Grundy SM, Brewer HB Jr, Cleeman JI, Smith SC Jr, Lenfant C; National Heart, Lung, and Blood Institute, et al. Definition of metabolic syndrome: report of the National Heart, Lung, and Blood Institute/American Heart Association conference on scientific issues related to definition. Arterioscler Thromb Vasc Biol 2004;24:e13-8.

2. Katsa ME, Ioannidis A, Zyga S, Tsironi M, Koutsovitis P, Chatzipanagiotou $S$, et al. The effect of nutrition and sleep habits on predisposition for metabolic syndrome in Greek children. J Pediatr Nurs 2018;40:e2-8.

3. Maruyama C, Imamura K, Teramoto T. Assessment of LDL particle size by triglyceride/HDL-cholesterol ratio in non-diabetic, healthy subjects without prominent hyperlipidemia. J Atheroscler Thromb 2003;10:186-91.

4. Toth PP. High-density lipoprotein and cardiovascular risk. Circulation 2004;109:1809-12.

5. González-Chávez A, Simental-Mendía LE, ElizondoArgueta S. Elevated triglycerides/HDL-cholesterol ratio associated with insulin resistance. Cir Cir 2011;79:126-31.

6. Johnson RJ, Kang DH, Feig D, Kivlighn S, Kanellis J, Watanabe $\mathrm{S}$, et al. Is there a pathogenetic role for uric acid in hypertension and cardiovascular and renal disease? Hypertension 2003;41:1183-90.

7. Sautin YY, Nakagawa T, Zharikov S, Johnson RJ. Adverse effects of the classic antioxidant uric acid in adipocytes: NADPH oxidase-mediated oxidative/nitrosative stress. Am J Physiol Cell Physiol 2007;293:C584-96.

8. Soltani Z, Rasheed K, Kapusta DR, Reisin E. Potential role of uric acid in metabolic syndrome, hypertension, kidney injury, and cardiovascular diseases: is it time for reappraisal? Curr Hypertens Rep 2013;15:175-81.

9. Zimmet P, Alberti G, Kaufman F, Tajima N, Silink M, Arslanian S, et al. The metabolic syndrome in children and adolescents. Lancet 2007;369:2059-61.

10. Liang J, Fu J, Jiang Y, Dong G, Wang X, Wu W. TriGlycerides and high-density lipoprotein cholesterol ratio compared with homeostasis model assessment insulin resistance indexes in screening for metabolic syndrome in the chinese obese children: a cross section study. BMC Pediatr 2015; 15:138.

11. Iwani NA, Jalaludin MY, Zin RM, Fuziah MZ, Hong JY, Abqariyah Y, et al. Triglyceride to HDL-C ratio is associated with insulin resistance in overweight and obese children. Sci Rep 2017;7:40055.

12. Yoo DY, Kang YS, Kwon EB, Yoo EG. The triglyceride-tohigh density lipoprotein cholesterol ratio in overweight Korean children and adolescents. Ann Pediatr Endocrinol Metab 2017;22:158-63.

13. Ford ES, Li C, Cook S, Choi HK. Serum concentrations of uric acid and the metabolic syndrome among US children and adolescents. Circulation 2007;115:2526-32.

14. Sun D, Li S, Zhang X, Fernandez C, Chen W, Srinivasan SR, et al. Uric acid is associated with metabolic syndrome in children and adults in a community: the Bogalusa Heart Study. PLoS One 2014;9:e89696.

15. Sun HL, Pei D, Lue KH, Chen YL. Uric acid levels can predict metabolic syndrome and hypertension in adolescents: a 10-year longitudinal study. PLoS One 2015;10:e0143786.

16. Perez ES, Medina MAG, Lomeli ML, González VT, Pérez JZV, Lavalle González FJ, et al. Association between serum uric acid and metabolic syndrome components in prepubertal obese children (Tanner Stage I) from Nuevo León, Mexico - a preliminary study. BMC Obes 2017;4:25.

17. Genoni G, Menegon V, Secco GG, Sonzini M, Martelli $\mathrm{M}$, Castagno $\mathrm{M}$, et al. Insulin resistance, serum uric acid and metabolic syndrome are linked to cardiovascular dysfunction in pediatric obesity. Int J Cardiol 2017;249:36671.

18. McCarthy HD, Jarrett KV, Crawley HF. The development of waist circumference percentiles in British children aged 5.0-16.9 y. Eur J Clin Nutr 2001;55:902-7.

19. Cen QJ, Lai HM, Chen BD, Li XM, Zhai H, He CH, et al. Appropriate LDL-C-to-HDL-C ratio cutoffs for categorization of cardiovascular disease risk factors among Uygur Adults in Xinjiang, China. Int J Environ Res Public Health 2016;13:235.

20. Atabek ME, Pirgon O, Kurtoglu S. Prevalence of metabolic syndrome in obese Turkish children and adolescents. Diabetes Res Clin Pract 2006;72:315-21.

21. Calcaterra V, De Giuseppe R, Biino G, Mantelli M, Marchini S, Bendotti G, et al. Relation between circulating oxidizedLDL and metabolic syndrome in children with obesity: the role of hypertriglyceridemic waist phenotype. J Pediatr Endocrinol Metab 2017;30:1257-63.

22. Rodrigues LG, Mattos AP, Koifman S. Prevalence of metabolic syndrome in overweight and obese outpatient children and adolescents: comparative analysis using 
different clinical definitions. Rev Paul Pediatr 2011;29:17885.

23. Emmanuel M, Bokor BR. Tanner Stages. StatPearls [Internet]. Treasure Island (FL): StatPearls Publishing; 2018 [cited 2018 Oct 27]. Available from: https://www.ncbi.nlm. nih.gov/books/NBK470280.

24. Parent AS, Teilmann G, Juul A, Skakkebaek NE, Toppari J, Bourguignon JP. The timing of normal puberty and the age limits of sexual precocity: variations around the world, secular trends, and changes after migration. Endocr Rev 2003;24:668-93.

25. da Luz PL, Favarato D, Faria-Neto JR Jr, Lemos P, Chagas AC. High ratio of triglycerides to HDL-cholesterol predicts extensive coronary disease. Clinics (Sao Paulo) 2008;63:427-32.

26. Dobiásová M. Atherogenic index of plasma [ $\log ($ triglycerides/HDL-cholesterol)]: theoretical and practical implications. Clin Chem 2004;50:1113-5.

27. Pacifico L, Bonci E, Andreoli G, Romaggioli S, Di Miscio $\mathrm{R}$, Lombardo CV, et al. Association of serum triglycerideto-HDL cholesterol ratio with carotid artery intima-media thickness, insulin resistance and nonalcoholic fatty liver disease in children and adolescents. Nutr Metab Cardiovasc Dis 2014;24:737-43.

28. Pacifico L, Nobili V, Anania C, Verdecchia P, Chiesa C. Pediatric nonalcoholic fatty liver disease, metabolic syndrome and cardiovascular risk. World J Gastroenterol 2011;17:3082-91.
29. Peng TC, Wang CC, Kao TW, Chan JY, Yang YH, Chang YW, et al. Relationship between hyperuricemia and lipid profiles in US adults. Biomed Res Int 2015;2015:127596.

30. Vekic J, Jelic-Ivanovic Z, Spasojevic-Kalimanovska V, Memon L, Zeljkovic A, Bogavac-Stanojevic N, et al. High serum uric acid and low-grade inflammation are associated with smaller LDL and HDL particles. Atherosclerosis 2009;203:236-42.

31. Lin SD, Tsai DH, Hsu SR. Association between serum uric acid level and components of the metabolic syndrome. J Chin Med Assoc 2006;69:512-6.

32. Baliarsingh S, Sharma N. Serum uric acid level is an indicator of total cholesterol and low density lipoprotein cholesterol in men below 45 years in age but not older males. Clin Lab 2012;58:545-50.

33. Afzali A, Weiss NS, Boyko EJ, Ioannou GN. Association between serum uric acid level and chronic liver disease in the United States. Hepatology 2010;52:578-89.

34. Ahrens W, Moreno LA, Mårild S, Molnár D, Siani A, De Henauw $S$, et al. Metabolic syndrome in young children: definitions and results of the IDEFICS study. Int J Obes (Lond) 2014;38 Suppl 2:S4-14.

35. Schwimmer JB, Deutsch R, Kahen T, Lavine JE, Stanley C, Behling C. Prevalence of fatty liver in children and adolescents. Pediatrics 2006;118:1388-93.

36. Alterio A, Alisi A, Liccardo D, Nobili V. Non-alcoholic fatty liver and metabolic syndrome in children: a vicious circle. Horm Res Paediatr 2014;82:283-9. 